\title{
Influence of the tip in near-field imaging of nanoparticle plasmonic modes: Weak and strong coupling regimes
}

\author{
Aitzol García-Etxarri, ${ }^{1,2}$ Isabel Romero, ${ }^{2}$ F. Javier García de Abajo, ${ }^{3}$ Rainer Hillenbrand, ${ }^{4}$ and Javier Aizpurua ${ }^{1,2, *}$ \\ ${ }^{1}$ Centro de Física de Materiales, CSIC-UPV/EHU, Paseo Manuel de Lardizabal 4, 20018 Donostia-San Sebastián, Spain \\ ${ }^{2}$ Donostia International Physics Center (DIPC), Paseo Manuel de Lardizabal 4, 20018 Donostia-San Sebastián, Spain \\ ${ }^{3}$ Instituto de Óptica-CSIC, Serrano 121, 28006 Madrid, Spain \\ ${ }^{4}$ CIC NanoGUNE Consolider, P. Mikeletegi 56, 20009 Donostia-San Sebastián, Spain
}

(Received 28 January 2009; published 30 March 2009)

\begin{abstract}
We identify weak and strong coupling regimes between a near-field probing tip and a plasmonic sample by imaging plasmon-resonant gold nanodisks with scattering-type scanning near-field optical microscopy (sSNOM). By means of rigorous electrodynamical calculations based on a model system, we find that in the weak coupling regime, s-SNOM can be applied for direct mapping of plasmonic nanoantenna modes, while in the strong coupling regime, the near-field probe allows for high-precision opto-mechanical control of the antenna response.
\end{abstract}

DOI: 10.1103/PhysRevB.79.125439

PACS number(s): 73.20.Mf, 78.67.-n

Surface plasmons in metallic nanoparticles have become a powerful driving force in the scientific and technological development of nanooptics. ${ }^{1-3}$ The ability of plasmons to act as the interface between far-field radiation and nanoscale confined near fields has generated promising prospects in plasmon-enhanced spectroscopy, ${ }^{4}$ optical nanoimaging, 5 electromagnetic signal guiding,, 6 and biosensing applications, ${ }^{8,9}$ among others. The potential of surface plasmons to decay radiatively, as well as nonradiatively, depending on the particular conformation and environment around them is the basis for most of these applications. In spite of the general and straightforward techniques available to obtain information on the far-field radiation by a surface plasmon such as in dark-field optical spectroscopy, ${ }^{10}$ the amplitude and phase of its near field is still more than a challenge to access. Electron-energy-loss spectroscopy for example has provided a tool for spatial mapping of the plasmon modes with unprecedent resolution, ${ }^{11}$ but it only provides information on the amplitude of the modes. However, in nanophotonics it is often the local near-field phase that is of extreme importance, as for example in coherent control applications, ${ }^{12}$ in nanoantenna-assisted molecular emission ${ }^{13}$ and spectroscopy, ${ }^{14}$ and in plasmon dynamics of complex metallic systems. ${ }^{15}$ A promising method to access both local amplitude and phase relies on near-field optical methods that have progressively succeeded in imaging nanoscale field patterns in metallic particles (optical nanoantennas). ${ }^{16-18}$

In particular, scattering-type scanning near-field optical microscopy (s-SNOM) (Ref. 19) has managed to map both the local amplitude and phase of the plasmon modes by interferometric detection of the antenna fields scattered by a scanning atomic force microscope tip. ${ }^{20-24}$ However, the antenna optical response is extremely sensitive to environmental changes, ${ }^{5,8,9}$ thus the process of measurement of its near field may result in the modification of the antenna modes, similar to probe-induced modifications in other nanophotonic systems. ${ }^{25-28}$ In this work we address this issue, presenting a basic understanding of the near-field coupling between s-SNOM probes and plasmonic nanoantennas (here gold nanodisks). We find that weak dielectric probes allow for plasmon mode mapping, whereas metallic probes introduce substantial modification of the antenna modes due to strong probe-antenna coupling. Furthermore, we explore the use of this strong coupling to locally and nanomechanically control the plasmon fields.

We provide experimental evidence of weak and strong coupling regimes in near-field probing of plasmonic nanoantennas by s-SNOM mapping of gold nanodisks at their resonance wavelength $\lambda=633 \mathrm{~nm}$ (Ref. 21). The s-SNOM used in our experiment [Fig. 1(a)] is based on an atomic force

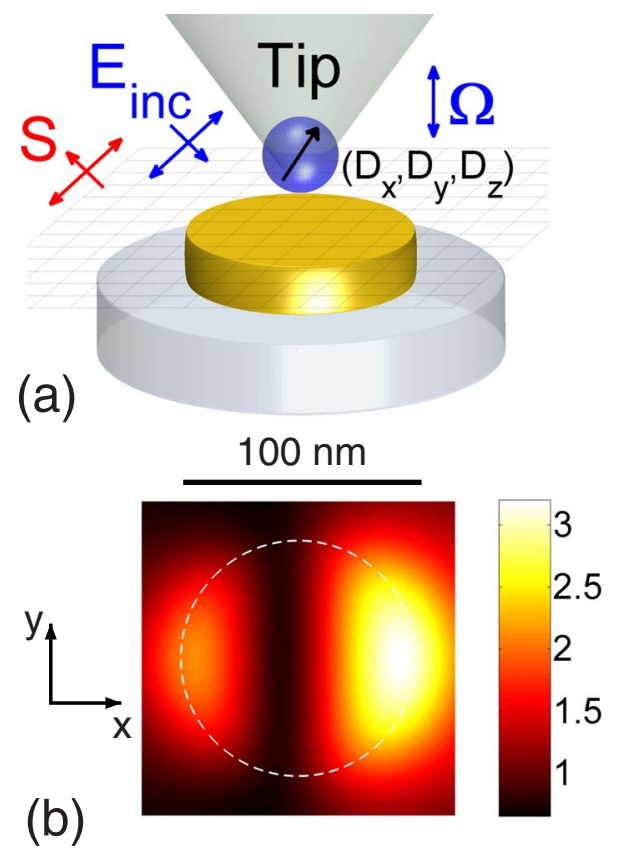

FIG. 1. (Color online) (a) Schematics of the near-field imaging process. A tip located at position $\left(D_{x}, D_{y}, D_{z}\right)$ scans the nanoantenna under study (gold nanodisk). We define $D_{x}=0, D_{y}=0$, and $D_{z}=0$ to be at the center of the disk on the top surface. Incident light $E_{\text {inc }}$ illuminates the tip-antenna system and backscattered far-field amplitude $s$ is recorded. (b) Calculated near-field amplitude on top of a gold nanodisk in absence of the tip. 


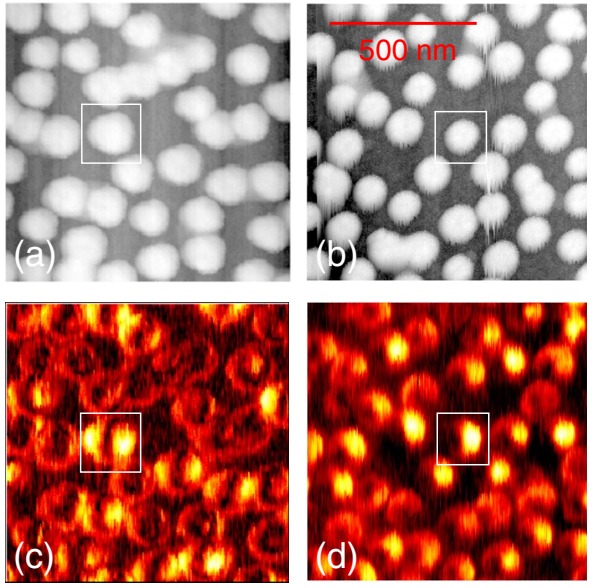

FIG. 2. (Color online) [(a) and (b)] Topography and [(c) and (d)] near-field images of a $1 \times 1$ micrometer size sample area showing several gold nanodisks. The images on the [(a) and (c)] left-hand side are topography and near field of disks taken with a carbon nanotube tip, whereas in the images of the $[(b)$ and (d)] right-hand side the topography and near field are obtained with a Pt-coated $\mathrm{Si}$ tip. Signals from the single disks marked by the white squares are represented in more detail in Fig. 3.

microscope (AFM) with both disk and tip being illuminated from the side with $P$-polarized He-Ne laser light. ${ }^{29}$ The measurement consists in the detection of light scattered from the tip that scans the nanodisk antennas. The AFM is operated in tapping mode where the tip is vibrating mechanically in $z$ direction with frequency $\Omega \approx 300 \mathrm{kHz}$ and an amplitude of $\approx 20 \mathrm{~nm}$, thus demodulation of the scattered far field allows for suppression of background scattering and for recovering the near-field signal. Heterodyne interferometric detection yields amplitude $s_{n}$ and phase $\varphi_{n}$ signals with $n$ being the $n$th harmonic of the tapping frequency $\Omega$.

The sample used in our experiments consists of randomly distributed nanodisks fabricated by colloidal lithography on glass. Because of the fabrication process, the disks present slight inhomogeneities in shape and size, therefore some of them departing from the average resonance at the wavelength of $633 \mathrm{~nm} .{ }^{21}$ From the images in Figs. 2(c) and 2(d), it can be clearly seen that the bright disks (those in resonance at the imaging wavelength of $633 \mathrm{~nm}$ ) exhibit a consistent nearfield pattern. Even though some of the particles present slightly different size and shape, and also different interparticle spacing, the bright patterns in resonance are consistent in an extended area of several $\mu \mathrm{m}^{2}$. This lets us conclude that the near-field pattern is not significantly affected by these parameters and that the near-field coupling between the disks is negligible. Note at this point that the tips used in the experiments have an apex radius of about $20-30 \mathrm{~nm}$, i.e., the distance between the particles is larger than seen in the images. Actually, the range of average separation between the disks is large enough to prevent relevant coupling between the particles, as derived from full electromagnetic calculations in similar situations. ${ }^{30}$

The absence of significant interparticle coupling allows us for studying the near-field patterns at a single individual nanodisk. In Figs. 3(a) and 3(b) we show topography and
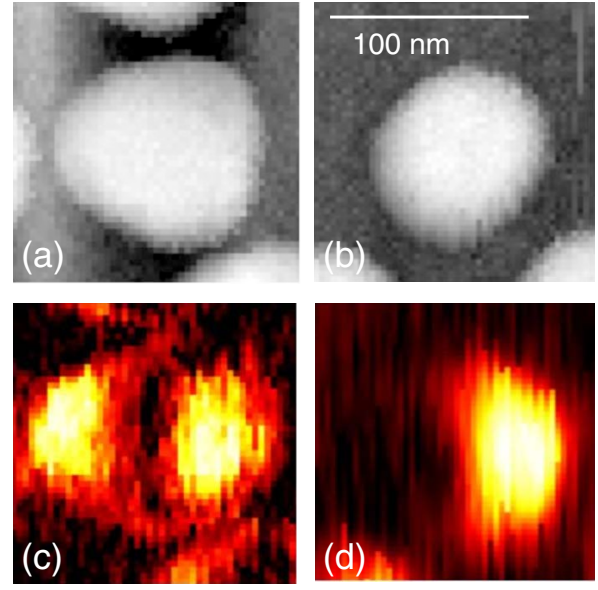

FIG. 3. (Color online) [(a) and (c)] Topography and near-field amplitude signal $s_{3}$ of a gold nanodisk obtained with use of a CNT tip. [(b) and (d)] Topography and near-field amplitude signal $s_{3}$ obtained with use of a Pt-coated Si tip.

background-free near-field amplitude images $s_{3}$ for the single nanodisks marked in Fig. 2, obtained with two different AFM tips: a carbon nanotube (CNT) [(a) and (c)] and a Ptcoated Si tip [(b) and (d)]. Using the CNT tip, the dipolar mode of the disk plasmon is clearly revealed in Fig. 3(c), as described in Ref. 21. The experimental image shows good agreement with the near-field amplitude calculated $21 \mathrm{~nm}$ above such a gold nanodisk in absence of the tip [Fig. 1(b)]. Note that the slight asymmetry in the field pattern is due to the illumination geometry. In contrast, using a metallized AFM tip, the plasmon modes of the gold nanoantennas are strongly modified [Fig. 3(d)] and a clearly asymmetric pattern can be observed in the amplitude image: a bright spot appears in the far edge of the disk with respect to the direction of the incoming radiation whereas a dark area is present in the near edge. In light of these results, we can conclude that under the present illumination/detection scheme, a metal tip strongly disturbs the near-field modes of plasmonic nanoantennas, thus preventing near-field optical mode mapping. With the weak tip, however, mode mapping is possible.

To understand the role of a probe in near-field imaging of plasmonic nanoantennas, we perform full electromagnetic calculations of the far-field amplitude $s$ backscattered by the probe-nanodisk system as a function of probe position with use of the boundary element method. ${ }^{31,32}$ For fundamental and straightforward insights into the interaction, we describe the probe as a point dipole with polarizability $\alpha_{\text {probe }}$ $=4 \pi R^{3}\left(\varepsilon_{\text {probe }}-1\right) /\left(\varepsilon_{\text {probe }}+2\right)$, associated with the polarizability of a virtual sphere of radius $R=32 \mathrm{~nm}$ and dielectric constant $\varepsilon_{\text {probe }}$. We use dielectric values for the probe of $\varepsilon_{\text {probe }}=1.5$ to resemble a weak dielectric probe (weak scatterer) and $\varepsilon_{\text {probe }}=-10+19 i$ to resemble a metallic Pt probe (strong scatterer). The calculation is performed for the resonance wavelength of the disk plasmon, $\lambda=633 \mathrm{~nm}$. $P$-polarized incident light and $P$-polarized detected light are considered under an incidence from the left of the disk (nearedge) of $45^{\circ}$. In Figs. 4(a) and 4(b) we show the calculated images of the backscattered far-field amplitude $s$ when scanning a probe dipole with low (a) and large (b) polarizabilities 

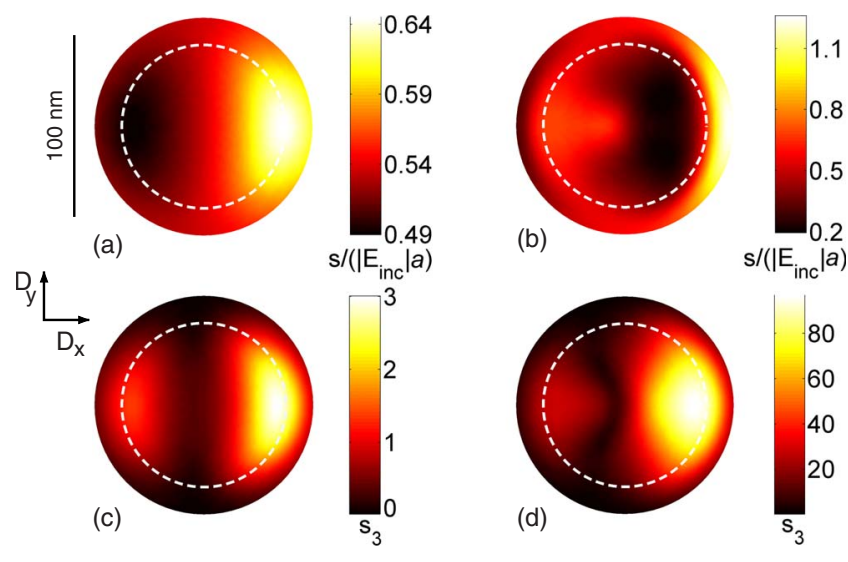

FIG. 4. (Color online) Calculated optical images of a 90-nmwide, 20-nm-high gold nanodisk at a wavelength of $\lambda=633 \mathrm{~nm}$. [(a) and (b)] Images showing the backscattered far-field amplitude $s$ normalized to the incident field $E_{\text {inc }}$ and the radius of the disk $a$, when the disk is scanned with (a) a weak dielectric probe and (b) a metallic probe. [(c) and (d)] Images showing the near-field signal $s_{3}$ when the disk is scanned with (c) a weak dielectric probe and (d) a metallic probe. The contours of the disks are marked by white dashed circles.

at constant height $D_{z}=21 \mathrm{~nm}$. The signal for a weak probe dipole [Fig. 4(a)] shows a nearly constant value with a slight increase at the far edge of the nanodisk (variation of 20\%). In this case, the disk scatters a field which is much stronger than the field scattered by the probe dipole, and therefore the total far-field amplitude $s$ is independent of the probe position. When scanning the probe, its weak far field is added to the nearly constant scattering of the disk. For a strong probe dipole [Fig. 4(b)], the strong near-field coupling between probe and nanodisk produces a far-field scattering which is more complex and strongly dependent on the position of the probe, yielding a significantly different pattern and large contrast. It is interesting to note that none of the images based on the far-field scattering (a) and (b) reveal the near-field modes of the disk.

To describe the experimental near-field images in Figs. 3(c) and 3(d) we need to account in our calculations for the modulation of the tip-sample distance. For each horizontal position of the probe on the disk $\left(D_{x}, D_{y}\right)$ we therefore calculate the backscattered far field $s$ as a function of the height of the probe $D_{z}$. Assuming a sinusoidal vertical motion of the tip with an amplitude equal to the experimental tapping amplitude $(20 \mathrm{~nm})$, we demodulate $s$ at the required order $n$. In our case we choose an order $n=3$ but other demodulation orders give similar results. Interestingly, the calculated $s_{3}$ images obtained for a weakly scattering probe [Fig. 4(c)] clearly reveal the dipolar near-field pattern of the disk [Fig. 1(b)]. Obviously, it is the demodulation procedure that allows us to recover the near-field distribution of the disk, which would be otherwise masked by the strong antenna far-field scattering [Fig. 4(a)]. Comparing now experiment and theory, we find a good agreement between the images $s_{3}$ recorded with a CNT tip [Fig. 3(c)] and the near-field signal calculated for a weakly scattering probe [Fig. 4(c)]. When we adopt a strongly scattering probe in our calculations, however, we find a much more pronounced bright spot in the
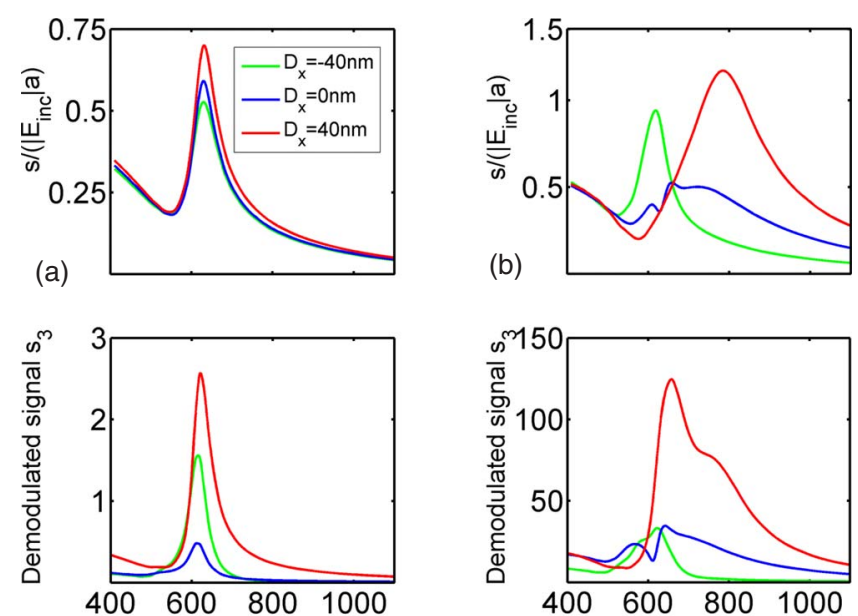

(c)
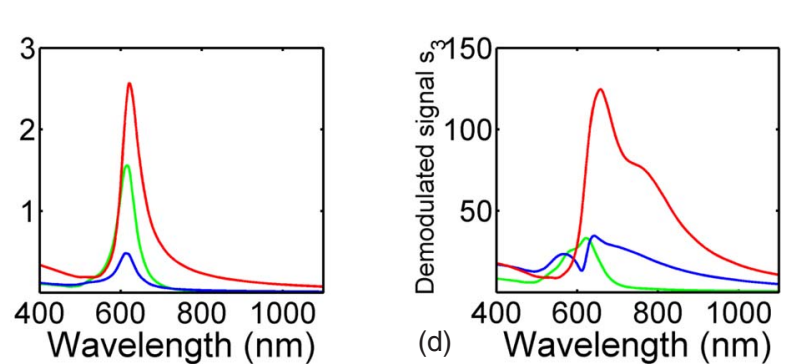

FIG. 5. (Color online) [(a) and (b)] Spectra of the backscattered far-field amplitude $s$ calculated for the probe-disk system normalized to the incident field $E_{\text {inc }}$ and the radius of the disk $a$. (a) Weak dielectric probe, (b) metallic probe. The vertical distance between probe and gold nanodisk is $D_{z}=21 \mathrm{~nm}$. [(c) and (d)] Spectra of the near-field signal $s_{3}$ calculated for (c) a weak dielectric probe and for (d) a metallic probe. Three different horizontal probe positions are considered in all calculations: far edge $D_{x}=+40 \mathrm{~nm}$ in red, center $D_{x}=0 \mathrm{~nm}$ in blue, and near edge $D_{x}=-40 \mathrm{~nm}$ in green.

far edge of the disk [Fig. 4(d)] indicating a distorted nearfield distribution. We actually find that the theoretical nearfield images [Fig. 4(d)] exhibit a distortion resembling the experimental maps obtained with a Pt tip [Fig. 3(d)], which do not reveal the antenna dipolar mode. We thus conclude the existence of both weak and strong coupling regimes between near-field probes and plasmonic nanoantennas.

To analyze the optical image contrasts in more detail, we study the spectral behavior of the backscattered amplitude $s$ [Figs. 5(a) and 5(b)] and of the near-field signals $s_{3}$ [Figs. $5(\mathrm{c})$ and 5(d)] for three relevant positions of the probe on top of the disk. The far edge $\left(D_{x}=+40 \mathrm{~nm}\right)$, center $\left(D_{x}\right.$ $=0 \mathrm{~nm})$, and near edge $\left(D_{x}=-40 \mathrm{~nm}\right)$ are selected as representative probe positions. For a weakly scattering probe [Figs. 5(a) and 5(c)], the position of the resonances in the backscattered amplitude $s$ and the near-field signal $s_{3}$ match well with the plasmon resonance of the disk $(\lambda=633 \mathrm{~nm})$. The presence of the tip obviously does not modify the spectral response of the nanoantenna, thus confirming weak coupling regime between the tip and the antenna. However, the amplitude $s$ is mostly independent of the probe position [see Fig. 5(a)], thus not revealing the dipolar near-field distribution when mapping the disk [see Fig. 4(a)]. The $s_{3}$ signal, in contrast, clearly reveals the position-dependent spectral behavior of the nanoantenna near field and thus allowing for plasmon mode mapping at $\lambda=633 \mathrm{~nm}$ [see Fig. 4(c)]. For a strongly scattering probe [Figs. 5(b) and 5(d)] we find considerable spectral shifts both in the far-field amplitude $s$ and in the near-field signal $s_{3}$, depending on the probe position. These spectral shifts provide clear evidence of strong tipantenna coupling modifying the plasmonic modes. The plasmon response is redshifted when the probe is located at the far edge of the disk (in red, dark gray) whereas a slight 

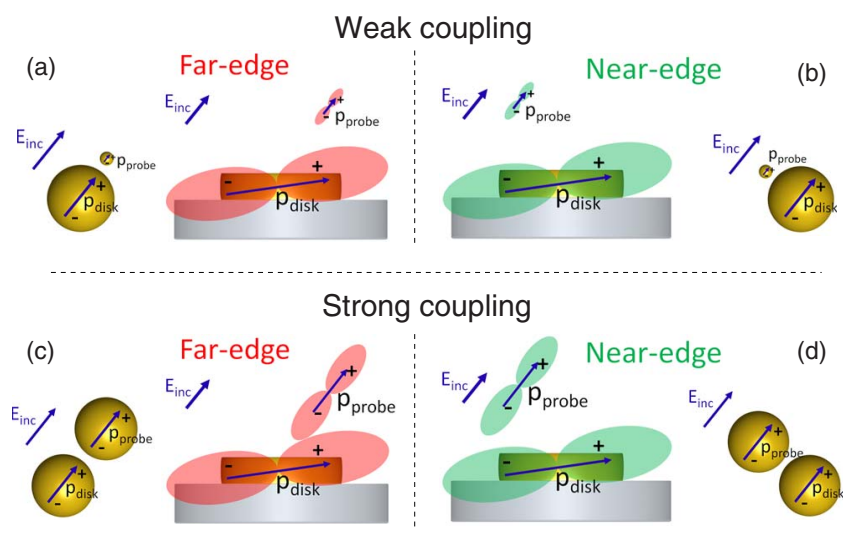

FIG. 6. (Color online) Schematics of the probe-disk coupling as a response to the incident field $E_{\text {inc }}$ in [(a) and (b)] weak and [(c) and (d)] strong probe-disk coupling regimes. When a weak probe dipole is located at the far edge (a) or the near edge (b) of a nanodisk, the total response is basically given by the disk by itself. (c) When a strong probe dipole is located at the far edge of a nanodisk, longitudinal probe-disk coupling is obtained. (d) When the strong probe dipole is located at the near edge of a nanodisk, transversal probe-disk coupling is generated. A schematics of the corresponding polarization pattern in terms of plasmonic particles is displayed close to each diagram.

blueshift is observed at the near-edge position (in green, light gray).

A simple understanding of the physics of such situations can be conceived in the schematics of Fig. 6 . The response of the disk and the tip can be interpreted as those corresponding to two dipoles characterized by different dipolar moments $\left(p_{\text {disk }}\right.$ and $\left.p_{\text {probe }}\right)$. In the case of a weakly interacting probe [Figs. 6(a) and 6(b)], the probe dipole $p_{\text {probe }}$ is weaker than the disk dipole $\left(p_{\text {disk }}\right)$, thus hardly affects the strong field produced by the disk dipole. In this case, the scattering is mainly given by the disk without distortion. However, in the strongly interacting regime [Figs. 6(c) and 6(d)], the polarizability of the scanning dipole $\alpha_{\text {probe }}$, and therefore its dipolar moment ( $\left.p_{\text {probe }}\right)$ is similar in strength, or even larger, than the dipole of the disk $\left(p_{\text {disk }}\right)$, therefore both dipoles interact very effectively, showing different spectral trends depending on their relative position. When the probe dipole is on the far edge of the disk $\left[D_{x}=+40 \mathrm{~nm}\right.$ in Fig. 6(c)], both dipoles are interacting mostly longitudinally, yielding a redshift of the response [see red line in Fig. 5(b)] similar to the longitudinal interaction of two metallic nanoparticles. ${ }^{30,33-35}$ When the tip is located at the near edge of the disk $\left[D_{x}=-40 \mathrm{~nm}\right.$ in Fig. 6(d)], both dipoles can be considered to be coupled mostly transversally, therefore showing a decrease in the near-field intensity with slight blueshift [see green line in Fig. 5(b)], similar to the case of transverse coupling of two nanoparticles. Essentially, for a strong probe dipole, it is this different probe-disk coupling scheme at the far edge compared to the near edge which is responsible for the spectral shifts and consequently for the distorted near-field images. This interpretation is fully justified in light of the experimental images of Fig. 3 and the numerical calculations in Fig. 4.

By modifying the near-field images of metallic nanoobjects, the strong probe-sample interaction introduces a tool to

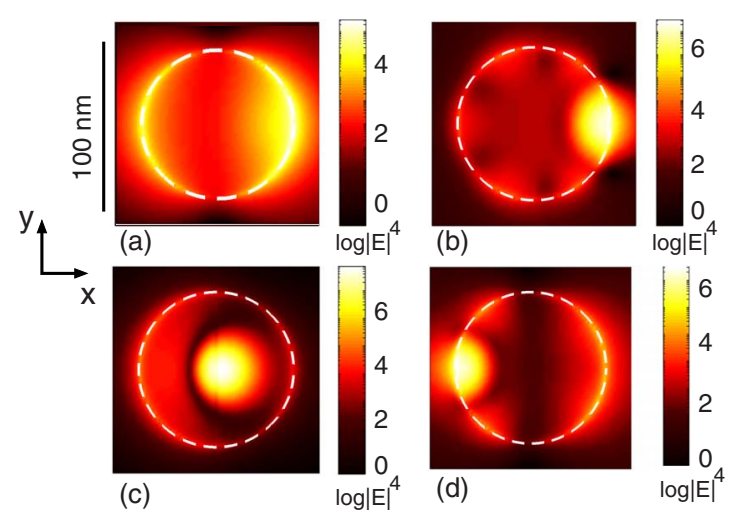

FIG. 7. (Color online) Fourth power of the calculated local field enhancement normalized to the incoming field on top of a gold nanodisk for four different positions of a strongly interacting dipolar probe: (a) far edge at $41 \mathrm{~nm}$ on top of the disk, (b) far edge at 21 $\mathrm{nm}$ on top of the disk, (c) center at $21 \mathrm{~nm}$ on top of the disk, and (d) near edge at $21 \mathrm{~nm}$ on top of the disk.

tune the optical response in plasmonic antennas and opens an avenue for a probe to become an active opto-mechanical element to control the field distribution and spatially activate selected areas of the nanoantenna with subnanometer precision. ${ }^{25}$ This tuning of the optical response is central for example in surface-enhanced spectroscopy ${ }^{33}$ and in molecular fluorescence near optical antennas. ${ }^{13}$ We show in Fig. 7 a set of near-field maps of the gold disk when a strongly interacting tip is located at four different positions. The near field is evaluated at $0.5 \mathrm{~nm}$ from the top surface of the nanodisk. The fourth power of the field enhancement $E=E_{\mathrm{loc}} / E_{\mathrm{inc}}$ is displayed to emphasize the importance of local enhancement in inelastic molecular spectroscopy where the field intensity of both incoming and outgoing fields is relevant for increasing the signal. Different vertical $D_{x}$ and horizontal $D_{z}$ probe positions generate very different near-field modes at the disk. When the probe is located at considerable vertical distance from the disk $\left(D_{z}=41 \mathrm{~nm}\right.$ at the far edge $)$ the field pattern shows the dipolar mode of the disk [Fig. 7(a)], similar to the near-field distribution calculated in absence of the tip [Fig. 1(b)], which permits to conclude that only negligible probeantenna coupling is present for such a distant positioning of the probe. As we get vertically closer to the disk $(21 \mathrm{~nm}$ on top of the disk surface), moving along the horizontal from the far edge [Fig. 7(b)], through the center [Fig. 7(c)], to the near edge [Fig. $7(\mathrm{~d})]$, the bright spot reveals the localization of the near field at the probe position. More interestingly, we find that higher order near-field modes are generated on the disk depending on the position of the probe $\left(D_{x}, D_{z}\right)$. Thus, the field enhancement at different areas of the plasmonic nanoantenna can be activated or deactivated by nanomechanical control of the tip-disk near-field interaction. This capability opens different perspectives, for example, in selective molecular light emission in the proximity of nanoantennas.

In summary, we have addressed quantitatively the influence of the tip in near-field imaging of plasmonic structures establishing the weak and strong near-field coupling regime between probe and antenna. Based on rigorous numerical 
calculations and on a simple interaction scheme, different near-field images of similar plasmonic disks can be correctly interpreted. Moreover, the strong-coupling regime introduces the capability for a near-field probe to control the optical response of a plasmonic antenna with a precision in the subnanometer range, which is otherwise not yet accessible with current fabrication technology. Both image interpretation and tuning capability are aspects of utmost importance in nanophotonics due to the prospects of ultrahigh-resolution amplitude and phase mapping of antenna modes, engineering of localized modes for selective plasmon mode activation, and sampling of nearby structures such as molecules probed via field-enhanced interaction.

We acknowledge P. Hanarp and D. S. Sutherland for provision of the disk samples, and financial support from the project ETORTEK-2008 of the Department of Industry of the Basque Government and project FIS2007-66711-C01-01 of the Spanish Ministry of Education and Science. *aizpurua@ehu.es

${ }^{1}$ W. L. Barnes, A. Dereux, and T. W. Ebbesen, Nature (London) 424, 824 (2003).

${ }^{2}$ H. A. Atwater, Sci. Am. 296, 56 (2007).

${ }^{3}$ M. Pelton, J. Aizpurua, and G. W. Bryant, Laser Photonics Rev. 2, 136 (2008).

${ }^{4}$ H. Xu, E. J. Bjerneld, M. Käll, and L. Borjesson, Phys. Rev. Lett. 83, 4357 (1999).

${ }^{5}$ L. Novotny and B. Hecht, Principles of Nano-optics (Cambridge University Press, Cambridge, 2006).

${ }^{6}$ S. A. Maier, P. G. Kik, H. A. Atwater, S. Meltzer, E. Harel, B. E. Koel, and A. A. G. Requicha, Nature Mater. 2, 229 (2003).

${ }^{7}$ S. I. Bozhevolnyi, V. S. Volkov, E. Devaux, J-Y. Laluet, and T. W. Ebbesen, Nature (London) 440, 508 (2006).

${ }^{8}$ G. Raschke, S. Kowarik, T. Franzl, C. Sönnichsen, T. Klar, J. Feldmann, A. Nichtl, and K. Kürzinger, Nano Lett. 3, 935 (2003).

${ }^{9}$ S. Lal, S. Link, and N. J. Halas, Nat. Photonics 1, 641 (2007).

${ }^{10}$ C. Sönnichsen, T. Franzl, T. Wilk, G. von Plessen, J. Feldmann, O. Wilson, and P. Mulvaney, Phys. Rev. Lett. 88, 077402 (2002).

${ }^{11}$ J. Nelayah et al., Nat. Phys. 3, 348 (2007).

${ }^{12}$ M. I. Stockman, S. V. Faleev, and D. J. Bergman, Phys. Rev. Lett. 88, 067402 (2002).

${ }^{13}$ T. H. Taminiau, F. D. Stefani, F. B. Segerink, and N. F. van Hulst, Nat. Photonics 2, 234 (2008).

${ }^{14}$ J. Aizpurua, T. Taubner, F. J. García de Abajo, M. Brehm, and R. Hillenbrand, Opt. Express 16, 1529 (2008).

${ }^{15}$ A. Kubo, K. Onda, H. Petek, Z. Sun, Y. S. Jung, and H. K. Kim, Nano Lett. 5, 1123 (2005).

${ }^{16}$ J. R. Krenn et al., Phys. Rev. Lett. 82, 2590 (1999).

${ }^{17}$ G. P. Wiederrecht, Eur. Phys. J. Appl. Phys. 28, 3 (2004).

${ }^{18}$ E. Cubukcu, E. A. Kort, K. B. Crozier, and F. Capasso, Appl. Phys. Lett. 89, 093120 (2006).
${ }^{19}$ F. Keilmann and R. Hillenbrand, Philos. Trans. R. Soc. London 362, 787 (2004).

${ }^{20}$ R. Hillenbrand and F. Keilmann, Appl. Phys. B: Lasers Opt. 73, 239 (2001)

${ }^{21}$ R. Hillenbrand, F. Keilmann, P. Hanarp, D. S. Sutherland, and J. Aizpurua, Appl. Phys. Lett. 83, 368 (2003).

${ }^{22}$ Z. H. Kim and S. R. Leone, Opt. Express 16, 1733 (2008).

${ }^{23}$ M. Rang, A. C. Jones, F. Zhou, Z-Y. Li, B. J. Wiley, Y. Xia, and M. B. Raschke, Nano Lett. 8, 3357 (2008).

${ }^{24}$ R. Esteban, R. Vogelgesang, J. Dorfmüller, A. Dmitriev, C. Rockstuhl, C. Etrich, and K. Kern, Nano Lett. 8, 3155 (2008).

${ }^{25}$ A. F. Koenderink, M. Kafesaki, B. C. Buchler, and V. Sandoghdar, Phys. Rev. Lett. 95, 153904 (2005).

${ }^{26}$ W. C. L. Hopman, K. O. van der Werf, A. J. F. Hollink, W. Bogaerts, V. Subramaniam, and R. M. de Ridder, Opt. Express 14, 8745 (2006).

${ }^{27}$ M. Achermann et al., Opt. Lett. 32, 2254 (2007).

${ }^{28}$ F. Intonti et al., Phys. Rev. B 78, 041401(R) (2008).

${ }^{29}$ R. Hillenbrand and F. Keilmann, Phys. Rev. Lett. 85, 3029 (2000).

${ }^{30}$ I. Romero, J. Aizpurua, G. W. Bryant, and F. J. García de Abajo, Opt. Express 14, 9988 (2006).

${ }^{31}$ F. J. García de Abajo and A. Howie, Phys. Rev. Lett. 80, 5180 (1998).

${ }^{32}$ F. J. García de Abajo and A. Howie, Phys. Rev. B 65, 115418 (2002).

${ }^{33}$ H. Xu, J. Aizpurua, M. Käll, and P. Apell, Phys. Rev. E 62, 4318 (2000).

${ }^{34}$ P. Nordlander, C. Oubre, E. Prodan et al., Nano Lett. 4, 899 (2004).

${ }^{35}$ J. B. Lassiter, J. Aizpurua, L. I. Hernandez, D. W. Brandl, I. Romero, S. Lal, J. H. Hafner, P. Nordlander, and N. J. Halas, Nano Lett. 8, 1212 (2008). 